\title{
Significant aspects and correlation between glycemic control and generalized chronic periodontitis in type 2 diabetes mellitus patients
}

\author{
MANUELA STOICESCU ${ }^{1 *}$, HOREA CALNICEANU ${ }^{2 *}$, IOAN ȚIG $^{3}$, SEBASTIAN NEMETH ${ }^{4}$, \\ ADRIANA TENT $^{3}$, ADELINA POPA $^{5 *}$, CIPRIAN BRISC $^{1 *}$ and IOANA IGNAT-ROMANUL ${ }^{3}$ \\ ${ }^{1}$ Department of Medical Disciplines, Faculty of Medicine and Pharmacy, University of Oradea, 410073 Oradea; \\ ${ }^{2}$ Department of Periodontology, 'Victor Babes' University of Medicine and Pharmacy of Timisoara, 300041 Timisoara; \\ ${ }^{3}$ Department of Dental Medicine, Faculty of Medicine and Pharmacy, University of Oradea, 410073 Oradea; \\ ${ }^{4}$ Pharmacy Department, Faculty of Medicine and Pharmacy, University of Oradea, 410028 Oradea; \\ ${ }^{5}$ Department of Orthodontics, 'Victor Babes' University of Medicine and Pharmacy of Timisoara, 300041 Timisoara, Romania
}

Received February 10, 2021; Accepted March 12, 2021

DOI: $10.3892 / \mathrm{etm} .2021 .10103$

\begin{abstract}
The purpose of this research was to assess the correlation between glycemic control and clinical periodontal characteristics in type 2 diabetics with generalized chronic periodontitis. A total of 182 patients with type 2 diabetes mellitus and generalized chronic periodontitis were included in our study. The clinical examination included full-mouth plaque accumulation, bleeding on probing (BoP), probing depth (PD), presence of suppuration (SUP), clinical attachment level (CAL) and number of remaining teeth. Blood analyses were conducted for glycated hemoglobin (HbAlc) and fasting plasma glucose (FPG). The correlation between the extent of periodontitis, defined as the percentage of PD and CAL sites $\geq 5 \mathrm{~mm}$, and glycemic control was also analyzed. In addition, clinical parameters were compared between two ( $<7$ and $\geq 7 \%$ ) glycemic subsets. The frequency of uncontrolled diabetic subjects $(\mathrm{HbA} 1 \mathrm{c} \geq 7 \%$ ) was higher than that of the well-controlled subjects (HbA1c <7\%), 57.15 vs. $42.85 \%$. Among the clinical parameters evaluated, mean full-mouth plaque accumulation was significantly higher among patients without glucose control $(74.2 \pm 25.2$ vs. $62.5 \pm 28.7 \%, \mathrm{P}<0.01)$,
\end{abstract}

Correspondence to: Dr Sebastian Nemeth, Pharmacy Department, Faculty of Medicine and Pharmacy, University of Oradea, 29 North Jiga Street, 410028 Oradea, Romania

E-mail: sebinemeth@yahoo.com

Dr Adriana Tent, Department of Dental Medicine, Faculty of Medicine and Pharmacy, University of Oradea, 1 Decembrie Square 10, 410073 Oradea, Romania

E-mail: adriana.tent@yahoo.com

*Contributed equally

Key words: HbA1c, periodontitis, type 2 diabetes, glycemic control, clinical examination as well as mean PD $(3.78 \pm 0.9$ vs. $3.42 \pm 0.8 \mathrm{~mm}, \mathrm{P}<0.01)$ and mean CAL ( $4.5 \pm 1.2$ vs. $4.1 \pm 1.2 \mathrm{~mm}, \mathrm{P}=0.02)$. The sites with $\mathrm{PD} \geq 5 \mathrm{~mm}$ were statistically more prevalent among patients with $\mathrm{HbA} 1 \mathrm{c} \geq 7 \%$ compared with patients with $\mathrm{HbA} 1 \mathrm{c}<7 \%$ $(27.8 \pm 6.2$ vs. $23.4 \pm 5.8 \%, \mathrm{P}<0.01)$. The mean number of remaining teeth was statistically significantly lower in patients with $\mathrm{HbA} 1 \mathrm{c} \geq 7 \%$ compared with patients with $\mathrm{HbA} 1 \mathrm{c}<7 \%$ $(18.5 \pm 3.2$ vs. $20.4 \pm 4.1, \mathrm{P}<0.01)$. In conclusion, the severity and extension of generalized chronic periodontitis was higher in type 2 diabetes mellitus patients with poor glucose control compared with those with good glucose control.

\section{Introduction}

Diabetes mellitus is a well-predicted risk factor for periodontal disease, and conversely, periodontitis is thought to affect the systemic inflammatory condition, insulin resistance, and lipid and glucose metabolism $(1,2)$. Being a chronic metabolic disease, it is caused by the body's failure either to produce the insulin hormone or to use insulin production effectively. Insulin is a hormone produced by the pancreas which enables the body to direct glucose from the bloodstream to cells for energy. Without this vital hormone, glucose builds up in the bloodstream and can lead to life-threatening and debilitating complications (3). Glycemic control is a key issue in the care of people with type 2 diabetes mellitus (T2DM). Hyperglycemia is the main cause of the progressive microvascular complications associated with diabetes (retinopathy, nephropathy, neuropathy) (4-9).

Periodontitis is a chronic inflammation of the tissues surrounding the teeth, due to the biofilm accumulation, in the absence of proper oral hygiene, that leads to formation of pockets between the teeth and gums, loss of periodontal support and bone [clinical attachment loss (CAL) and bone loss] which could lead to tooth mobility and subsequently the need to remove the teeth.

The biofilm is composed of more than 1,000 different species of microorganisms but some strictly anaerobic bacteria 
such as Porphyromonas gingivalis, Tannerella forsythia, Treponema denticola and Aggregatibacter atinomycetemcomitans are frequently associated with the initiation and perpetuation of periodontitis (10-12).

A bi-directional association between periodontitis and diabetes has been postulated (13). The relationship between diabetes and chronic periodontitis has recently attracted the attention of researchers worldwide. Many studies have suggested a two-way relationship between diabetes mellitus and chronic periodontitis. Over the years, epidemiological studies have consistently reported that increased incidence, prevalence and acuity of periodontitis is found among adults with T2DM (14). The incidence of periodontitis increases as diabetic patients age and is both more frequent and more severe in T2DM patients with advanced systemic complications (15). Poor glycemic control may be associated with an increased risk of periodontal disease or increased severity of periodontal disease in patients with diabetes. T2DM is therefore considered to be one of the risk factors for periodontal disease (16), and improving glycemic control can reduce the pathological progression of periodontal disease $(17,18)$. There is also a vice versa assumption that periodontitis is considered to increase the risk of developing diabetes mellitus (14).

Current scientific evidence suggests that better metabolic control brings significant benefits to the treatment of chronic periodontitis. In consequence, the main aim of the current research study was to assess the correlation between glycemic control and clinical periodontal aspects in patients with T2DM associated with generalized chronic periodontitis; moreover, some particular aspects regarding this association were followed, suggesting the possibility of a protocol that associates the treatment of chronic periodontal disease as a diagnostic criterion/follow-up of subjects with T2DM.

\section{Patients and methods}

This study was approved by the Ethics Commission of the Emergency Clinical County Hospital of Oradea, Oradea, Romania, and was conducted respecting the World Medical Association (WMA) Code of Ethics, Declaration of Helsinki 1967 (19). All patients were verbally informed, and they signed an informed consent form.

From the initial group of 279 patients, due to the selection criteria, 182 patients (age range, 40-68 years) diagnosed with T2DM and generalized chronic periodontitis completed the study and were evaluated in two private dental clinics in Oradea, Romania, from July 2018 to February 2020.

The inclusion criteria were as follows: i) $\geq 40$ yeas of age; ii) confirmed type 2 diabetes for at least 5 years; iii) diagnosis of chronic generalized periodontitis; iv) history of T2DM therapy.

The exclusion criteria were as follows: i) Smoker or tobacco use in any form, in the preceding 5 years; ii) pregnant or lactating; iii) mouth washing with antimicrobial agent in the past 2 months; iv) chronic disease, excluding DM, associated periodontal disease; $v$ ) periodontal or/and antibiotic therapy in the preceding 6 months.

The participants were divided into two groups, according to the determined HbAlc levels. Group 1 included patients with HbAc1 $<7 \%$ (considered to have a good glycemic control) and
Group 2 comprised of patients with $\mathrm{HbAc1} \geq 7 \%$ (considered to have a poor glycemic control).

Fasting blood glucose (FBG) from venous blood was determined using glucose oxidase method, expressed in milligrams per deciliter (mg/dl), while HbAlc was determined using high-performance liquid chromatography (HPLC) and expressed as a percentage. All the patients underwent a periodontal evaluation using a manual periodontal probe (PCP-UNC15; Hu Friedy). The periodontal parameters recorded were as follows: PI (plaque index), BoP (bleeding on probing), PD (probing depth) and CAL (clinical attachment level). The percentage of $\mathrm{PD} \geq 5 \mathrm{~mm}$ and $\mathrm{CAL} \geq 5 \mathrm{~mm}$ sites and the number of remaining teeth for each individual were also recorded.

From the point of view of statistical analysis, in order to examine the data distribution, the normality Kolmogorov-Smirnov test was used; also, because the data had a normal distribution ( $\mathrm{P}>0.05)$, analysis was performed using parametric methods. PD and CAL were dichotomized into $<5$ or $\geq 5 \mathrm{~mm}$. The full-mouth mean percentage of sites with visible plaque, BoP, $\mathrm{PD} \geq 5 \mathrm{~mm}, \mathrm{CAL} \geq 5 \mathrm{~mm}$ and SUP, the full-mouth mean PD and CAL were determined for each subject. The clinical parameters were also grouped into subsets of glycemia, according to two, $<7 \%$ (good glycemic control) and $\geq 7 \%$ (poor glycemic control) levels of HbAlc. Values for each clinical parameter were averaged separately within the two glycemic categories. The clinical parameters between HbA1c subsets $<7$ and $\geq 7 \%$ were compared by Student's t-test. The Chi-square test was used to detect differences in the frequencies of sex and DM treatment regimen among the glycemic groups. A value of $\mathrm{P}<0.05$ was considered statistically significant.

\section{Results}

A total of 279 patients were screened at two private dental clinics in Oradea, Romania from July 2018 to February 2020. The study was completed by 182 participants on the basis of inclusion/exclusion criteria, divided in two groups. The percentage of uncontrolled diabetic subjects (Group 2) was higher than that of the well-controlled subjects (Group 1) (57.15 vs. $42.85 \%$ ); uncontrolled diabetes being correlated with increased levels of HbAc1 and FPG. Age, sex, duration of DM, and diet did not have any statistical significance between the two groups; for the numerical variables we applied a Student t-test and for the qualitative variable we applied the Chi-square test. Data resulted are depicted in Table I.

The mean full-mouth plaque accumulation index (74.2 \pm 25.2 vs. $62.5 \pm 28.7 \%, \mathrm{P}<0.01)$, as well as the mean PD $(3.78 \pm 0.9$ vs. $3.42 \pm 0.8 \mathrm{~mm}, \mathrm{P}<0.01)$, and mean $\mathrm{CAL}$ $(4.5 \pm 1.2$ vs. $4.1 \pm 1.2 \mathrm{~mm}, \mathrm{P}=0.02)$ were significantly higher in Group 2 patients when compared with Group 1 patients. The sites with $\mathrm{PD} \geq 5 \mathrm{~mm}$ were statistically more prevalent in patients with $\mathrm{HbAlc} \geq 7 \%$ than that in patients with $\mathrm{HbAlc}<7 \%$ $(27.8 \pm 6.2$ vs. $23.4 \pm 5.8 \%, \mathrm{P}<0.01)$. The mean number of remaining teeth was statistically significantly lower in Group 2 patients $(18.5 \pm 3.2$ vs. $20.4 \pm 4.1, \mathrm{P}<0.01)$ than in that in Group 1 patients. For the numerical variables, a Student's t-test was applied and for the qualitative variable we used the Chi-square test. The results that were obtained are presented in Table II. 
Table I. Diabetes parameters.

\begin{tabular}{lccc}
\hline Parameters & HbA1c $<7 \%($ Group 1) & HbA1c $\geq 7 \%$ (Group 2) & P-value \\
\hline No. of subjects (\%) & $78(42.85 \%)$ & $104(57.15 \%)$ & $<3.7 \pm 7.1$ \\
Mean age (years) & $54.2 \pm 6.8$ & 49.03 & 0.63 \\
Sex (\% men) & 48.1 & $5.9 \pm 1.8$ & 0.9 \\
Duration of DM (years) & $6.3 \pm 1.5$ & 100 & 0.11 \\
Diet (\%) & 100 & 50.00 & $>0.05$ \\
Diet + oral hypoglycemic agents (\%) & 58.23 & 35.58 & 0.27 \\
Diet + insulin (\%) & 29.11 & 14.42 & 0.35 \\
Diet + insulin + oral hypoglycemic agents (\%) & 12.66 & $146 \pm 46.8$ & 0.73 \\
Mean FPG (mg/d) & $124 \pm 34.8$ & $<\mathbf{0 1}$
\end{tabular}

HbA1c, glycated hemoglobin; DM, diabetes mellitus; FPG, fasting plasma glucose. Statistically significant P-values are noted in bold print.

Table II. Periodontal parameters.

\begin{tabular}{lccc}
\hline Parameters & HbA1c $<7 \%$ (Group 1) & HbA1c $\geq 7 \%$ (Group 2) & P-value \\
\hline Mean full-mouth plaque accumulation (\%) & $62.5 \pm 28.7$ & $74.2 \pm 25.2$ & $<\mathbf{0 . 0 1}$ \\
Mean full-mouth PD (mm) & $3.42 \pm 0.8$ & $3.78 \pm 0.9$ & $4.5 \pm 1.2$ \\
Mean full-mouth CAL (mm) & $4.1 \pm 1.2$ & $47.90 \pm 30.1$ & $\mathbf{0 . 0 2}$ \\
Mean full-mouth BoP (\%) & $45.24 \pm 27.5$ & $3.1 \pm 2.8$ & 0.53 \\
Mean full-mouth SUP (\%) & $2.7 \pm 2.1$ & $27.8 \pm 6.2$ & 0.28 \\
Sites with PD $\geq 5$ mm (\%) & $23.4 \pm 5.8$ & $42.6 \pm 13.5$ & $<\mathbf{0 1}$ \\
Sites with CAL $\geq 5$ mm (\%) & $43.7 \pm 12.1$ & $18.5 \pm 3.2$ & 0.56 \\
Number of teeth (n) & $20.4 \pm 4.1$ & $<\mathbf{0 1}$
\end{tabular}

PD, probing depth; CAL, clinical attachment level; BoP, bleeding on probing; SUP, presence of suppuration. Statistically significant P-values are noted in bold print.

\section{Discussion}

Current literature suggests two-way inter-relationships between diabetes mellitus and periodontitis (20). Previous longitudinal studies have shown associations between baseline periodontitis and diabetes incidence (21-24) whereas one showed no association (25). Few cross-sectional studies showed periodontitis to be associated with precursors of diabetes (26-30). Consequently, since periodontitis-diabetes interactions may have a negative effect on patient glycemic control, the management of highly prevalent periodontal disease in diabetic patients is beneficial in improving overall health status.

The exact mechanisms by which glycemic control influences periodontal disease and its treatment may affect glycemic control, in patients with type 2 diabetes mellitus (T2DM), have not been fully elucidated. A popular hypothesis involves inflammatory pathways common to periodontitis and insulin resistance pathogenesis (20).

Botero et al showed that the reduction in HbA1c values (0.23 to $1.03 \%$ ) was significant for the treatment of T2DM (31). However, it was observed that for every percentage point decrease in HbAlc (e.g. 9 to $8 \%$ ), there was a $35 \%$ reduction in the risk of complications, with periodontal disease being a major complication of diabetes (32). Therefore, it is biologically plausible that periodontitis may exacerbate glycemia in T2DM patients, and that treatment may improve glycemic control by reducing circulating cytokine levels (33).

Preshaw et al and Winning et al reported similar results with our findings. Therefore, the number of poor glycemic control patients was higher than the number of good glycemic control patients. Increased values were found in poor glycemic control patients, when comparing the periodontal parameters of the two groups (PD, CAL, BoP and SUP) $(20,21)$.

The most striking changes in uncontrolled diabetes are the reduction in defense mechanism and the increased susceptibility to infections, leading to destructive periodontal disease. The glucose content of gingival fluid and blood is higher in individuals with diabetes than in those without diabetes, with similar plaque and GI scores (34). The increased glucose in the gingival fluid and blood of diabetic patients could change the environment of the microflora, inducing qualitative changes in bacteria that could contribute to the severity of periodontal disease observed in those with poorly controlled diabetes. In patients with poorly controlled diabetes, the function of polymorphonuclear granulocytes (PMNs) and monocytes/macrophages is impaired (35), and as a result, the primary defense (PMNs) against periodontal pathogens is diminished, and bacterial proliferation is more likely. 
The correlation between worsening periodontitis and poor glycemic control in the Group 2 patients was also reflected in the reduced number of teeth (mean value of 20.4 in Group 1 vs. 18.5 in Group 2). This result was in accordance with a study performed by Joshipura et al (13). The presence of periodontitis increases the risk of worsening of glycemic control over time. For example, in a 2-year longitudinal trial, diabetic subjects with severe periodontitis at baseline had a six-fold increased risk of worsening of glycemic control over time compared to diabetic subjects without periodontitis (36).

Group 2 patients had significantly worse periodontal status, deeper periodontal pocket and higher attachment loss. Increased bleeding on probing and suppuration index were also increased for Group 2 patients. These results are in concordance with the findings reported by $\mathrm{Wu}$ et al in a systematic review (37).

Periodontitis may also be associated with an increased risk of other diabetic complications, as seen in a longitudinal case-control study in which $82 \%$ of diabetic patients with severe periodontitis experienced the onset of one or more major cardiovascular, cerebrovascular, or peripheral vascular events compared to only $21 \%$ of diabetic subjects without periodontitis (13).

Although many diabetic patients show improvement in clinical parameters of disease immediately after therapy, patients with poorer glycemic control may have a more rapid recurrence of deep pockets and a less favorable long-term response (38).

As the present research proves once again by the results obtained, it is recommended that the criterion of inclusion of patients with periodontal treatment may be an important part of the protocol for the management of diabetic patients.

In conclusion, the severity and extension of generalized chronic periodontitis are higher in T2DM patients with poor glycemic control compared to patients with good glycemic control.

\section{Acknowledgements}

Not applicable.

\section{Funding}

No funding was received.

\section{Availability of data and materials}

Data of the patients are registered at the Clinical County Emergency Hospital of Oradea, Bihor, Romania and at the two private dental clinics, Oradea, Romania. Any further information concerning the study is available from the corresponding author upon reasonable request.

\section{Authors' contributions}

MS, HC, AT and IT made the interventions and collected, analyzed and interpreted the patient data regarding pre-intervention and post-intervention evolution. SN, AP, CB and IIR made substantial contributions to the conception of the work and interpretation of data; also, they drafted the manuscript and were major contributors in writing the manuscript. All authors read and approved the final manuscript to be published. All the authors agreed to be accountable for all aspects of the work in ensuring that questions related to the accuracy or integrity of any part of the work are appropriately investigated and resolved.

\section{Ethics approval and consent to participate}

This research was authorized by the Ethics Commission of the Emergency Clinical County Hospital of Oradea, Oradea, Romania, and conducted respecting the World Medical Association (WMA) Code of Ethics (Declaration of Helsinki 1967).

\section{Patient consent for publication}

Not applicable.

\section{Competing interests}

The authors declare that they have no competing interests.

\section{References}

1. Singh M, Bains VK, Jhingran R, Srivastava R, Madan R, Maurya SC and Rizvi I: Prevalence of periodontal disease in type 2 diabetes mellitus patients: A cross-sectional study. Contemp Clin Dent 10: 349-357, 2019.

2. Vesa CM, Popa L, Popa AR, Rus M, Zaha AA, Bungau S, Tit DM, Corb Aron RA and Zaha DC: Current data regarding the relationship between type 2 diabetes mellitus and cardiovascular risk factors. Diagnostics 10: 314, 2020.

3. Simpson TC, Weldon JC, Worthing ton HV, Needleman I, Wild SH, Moles DR, Stevenson B, Furness S and Iheozor-Ejiofor Z: Treatment of periodontal disease for glycaemic control in people with diabetes mellitus. Cochrane Database Syst Rev: Nov 6, 2015 (Epub ahead of print). doi: 10.1002/14651858.CD004714.pub3.

4. Grant RW and Kirkman MS: Trends in the evidence level for the American Diabetes Association's 'Standards of Medical Care in Diabetes' from 2005 to 2014. Diabetes Care 38: 6-8, 2015.

5. Dobrică EC, Găman MA, Cozma MA, Bratu OG, Pantea Stoian A and Diaconu CC: Polypharmacy in type 2 diabetes mellitus: Insights from an internal medicine department. Medicina (Kaunas) 55: 436, 2019.

6. Iorga RA, Bacalbasa N, Carsote M, Bratu OG, Stanescu AM, Bungau S, Pantis C and Diaconu CC: Metabolic and cardiovascular benefits of GLP-1 agonists, besides the hypoglycemic effect (Review). Exp Ther Med 20: 2396-400, 2020.

7. Diaconu C, Salmen T, Găman MA, Bratu OG, Mischianu D, Marcu RD, Suceveanu AI, Costache RS and Pantea Stoian A: SGLT2 inhibition in patients with type 2 diabetes and cardiovascular diseases: Which are the benefits? Rom J Mil Med 122: 16-21, 2019

8. Zaha CD, Vesa C, Uivarosan D, Bratu O, Fratila O, Tit DM, Pantis C, Diaconu CC and Bungau S: Influence of inflammation and adipocyte biochemical markers on the components of metabolic syndrome. Exp Ther Med 20: 121-128, 2020.

9. Popa AR, Fratila O, Rus M, Aron RA, Vesa CM, Pantis C, Diaconu CC, Bungau S and Nemeth S: Risk factors for adiposity in the urban population and influence on the prevalence of overweight and obesity. Exp Ther Med 20: 129-133, 2020.

10. Kumar PS: Oral microbiota and systemic disease. Anaerobe 24: 90-93, 2013.

11. Zaha DC, Bungau S, Aleya S, Tit DM, Vesa CM, Popa AR, Pantis C, Maghiar OA, Bratu OG, Furau C, et al: What antibiotics for what pathogens? The sensitivity spectrum of isolated strains in an intensive care unit. Sci Total Environ 687: 118-127, 2019.

12. Zaha DC, Bungau S, Uivarosan D, Tit DM, Maghiar TA, Maghiar O, Pantis C, Fratila O, Rus M and Vesa CM: Antibiotic consumption and microbiological epidemiology in surgery departments: Results from a single study center. Antibiotics Basel 9: 81, 2020. 
13. Joshipura KJ, Muñoz-Torres FJ, Dye BA, Leroux BG, Ramírez-Vick $M$ and Pérez CM: Longitudinal association between periodontitis and development of diabetes. Diabetes Res Clin Pract 141: 284-293, 2018.

14. Mealey BL and Oates TW: Diabetes mellitus and periodontal diseases. J Periodontol 77: 1289-1303, 2006.

15. Löe H: Periodontal disease. The sixth complication of diabetes mellitus. Diabetes Care 16: 329-334, 1993.

16. Jimenez M, Hu FB, Marino M, Li Y and Joshipura KJ: Type 2 diabetes mellitus and 20 year incidence of periodontitis and tooth loss. Diabetes Res Clin Pract 98: 494-500, 2012.

17. Katagiri S, Nitta H, Nagasawa T, Izumi Y, Kanazawa M, Matsuo A, Chiba H, Fukui M, Nakamura N, Oseko F, et al: Effect of glycemic control on periodontitis in type 2 diabetic patients with periodontal disease. J Diabetes Investig 4: 320-325, 2013.

18. Goyal L, Gupta ND and Bey A: Periodontal therapy: A useful adjunct to improve glycemic control. J Pharm Bioallied Sci 4: 173-174, 2012.

19. World Medical Association declaration of Helsinki. Recommendations guiding physicians in biomedical research involving human subjects. JAMA 277: 925-926, 1997.

20. Preshaw PM, Alba AL, Herrera D, Jepsen S, Konstantinidis A, Makrilakis K and Taylor R: Periodontitis and diabetes: A two-way relationship. Diabetologia 55: 21-31, 2012.

21. Winning L, Patterson CC, Neville CE, Kee F and Linden GJ: Periodontitis and incident type 2 diabetes: A prospective cohort study. J Clin Periodontol 44: 266-274, 2017.

22. Miyawaki A, Toyokawa S, Inoue K, Miyoshi Y and Kobayashi Y: Self-reported periodontitis and incident type 2 diabetes among male workers from a 5-year follow-up to MY Health Up Study. PLoS One 11: e0153464, 2016.

23. Chiu SY, Lai H, Yen AM, Fann JC, Chen LS and Chen HH Temporal sequence of the bidirectional relationship between hyperglycemia and periodontal disease: A community-based study of 5,885 Taiwanese aged 35-44 years (KCIS No. 32). Acta Diabetol 52: 123-131, 2015.

24. Demmer RT, Jacobs DR Jr and Desvarieux M: Periodontal disease and incident type 2 diabetes: Results from the First National Health and Nutrition Examination Survey and its epidemiologic follow-up study. Diabetes Care 31: 1373-1379, 2008.

25. Kebede TG, Pink C, Rathmann W, Kowall B, Völzke H, Petersmann A, Meisel P, Dietrich T, Kocher T and Holtfreter B: Does periodontitis affect diabetes incidence and haemoglobin A1c change? An 11-year follow-up study. Diabetes Metab 44: 243-249, 2008

26. Benguigui C, Bongard V, Ruidavets JB, Chamontin B, Sixou M, Ferrières J and Amar J: Metabolic syndrome, insulin resistance, and periodontitis: A cross-sectional study in a middle-aged French population. J Clin Periodontol 37: 601-608, 2010.
27. Demmer RT, Squillaro A, Papapanou PN, Rosenbaum M, Friedewald WT, Jacobs DR Jr and Desvarieux M: Periodontal infection, systemic inflammation, and insulin resistance: Results from the continuous National Health and Nutrition Examination Survey (NHANES) 1999-2004. Diabetes Care 35: 2235-2242, 2012.

28. Islam SK, Seo M, Lee YS and Moon SS: Association of periodontitis with insulin resistance, $\beta$-cell function, and impaired fasting glucose before onset of diabetes. Endocr J 62: 981-989, 2015.

29. Song IS, Han K, Park YM, Ji S, Jun SH, Ryu JJ and Park JB: Severe periodontitis is associated with insulin resistance in non-abdominal obese adults. J Clin Endocrinol Metab 101: 4251-4259, 2016.

30. Timonen P, Suominen-Taipale L, Jula A, Niskanen M, Knuuttila M and Ylöstalo P: Insulin sensitivity and periodontal infection in a non-diabetic, non-smoking adult population. J Clin Periodontol 38: $17-24,2011$

31. Botero JE, Rodríguez C and Agudelo-Suarez AA: Periodontal treatment and glycaemic control in patients with diabetes and periodontitis: An umbrella review. Aust Dent J 61: 134-148, 2016.

32. Genuth S, Eastman R, Kahn R, Klein R, Lachin J, Lebovitz H, Nathan D and Vinicor F; American Diabetes Association: Implications of the United Kingdom prospective diabetes study. Diabetes Care 26 (Suppl 1): S28-S32, 2003

33. DPTT study group; Engebretson S, Gelato M, Hyman L, Michalowicz BS and Schoenfeld E: Design features of the diabetes and periodontal therapy trial (DPTT): A multicenter randomized single-masked clinical trial testing the effect of nonsurgical periodontal therapy on glycosylated hemoglobin (HbAlc) levels in subjects with type 2 diabetes and chronic periodontitis. Contemp Clin Trials 36: 515-526, 2013

34. Ficara AJ, Levin MP, Grower MF and Kramer GD: A comparison of the glucose and protein content of gingival fluid from diabetics and nondiabetics. J Periodontal Res 10: 171-175, 1975.

35. Iacopino AM: Periodontitis and diabetes interrelationships: Role of inflammation. Ann Periodontol 6: 125-137, 2001.

36. Taylor GW, Burt BA, Becker MP, Genco RJ, Shlossman M, Knowler WC and Pettitt DJ: Severe periodontitis and risk for poor glycemic control in patients with non-insulin-dependent diabetes mellitus. J Periodontol 67: 1085-1093, 1996.

37. Wu CZ, Yuan YH, Liu HH, Li SS, Zhang BW, Chen W, An Z, Chen $\mathrm{S}, \mathrm{Wu} \mathrm{Y}$, Han B, et al: Epidemiologic relationship between periodontitis and type 2 diabetes mellitus. BMC Oral Health 20: 204, 2020.

38. Tervonen $\mathrm{T}$ and Karjalainen $\mathrm{K}$ : Periodontal disease related to diabetic status. A pilot study of the response to periodontal therapy in type 1 diabetes. J Clin Periodontol 24: 505-510, 1997. 